\title{
Further explorations with a process model for water jug problems
}

\author{
MICHAEL E. ATWOOD \\ Science Applications, Inc., Denver, Colorado 80111 \\ and \\ MICHAEL E. J. MASSON and PETER G. POLSON
University of Colorado, Boulder, Colorado 80309
}

\begin{abstract}
Subjects performed a water jug task in which the object was to find a sequence of pouring operations that would produce a specified amount of water in each jug. A model for this task is presented and evaluated. The model makes strong assumptions concerning lack of planning in the water jug task. In addition, alternative models incorporating planning assumptions were considered. Alternative models were evaluated in two experiments. All experimental conditions were successfully simulated by making reasonable and well-motivated parameter changes in our model, which assumes no planning. Further, several classes of models incorporating planning assumptions were shown to be inconsistent with the observed results.
\end{abstract}

In a previous paper (Atwood \& Polson, 1976), two of us developed a model for water jug problems. The purpose of this paper is to provide additional experimental support for the basic process assumptions underlying this model. In particular, we want to show that alternative theories assuming more complex moveselection processes cannot provide adequate descriptions of performance on water jug problems.

Water jug problems belong to a class of problems called transformation problems by Greeno (1978) and MOVE problems by Einst and Newell (1969). The primary characteristic of such problems is the generation of successor states from the current problem state by a single complex operator. Other examples of such problems include the Missionaries-Cannibals problem and the Towers of Hanoi.

Jeffries, Polson, Razran, and Atwood (1977) have shown that the framework underlying the Atwood and Polson (1976) model can be used to derive a successful simulation for the Missionaries-Cannibals and related river-crossing problems. Although this supports the generality of this framework, neither the Jeffries et al.

This research was undertaken within the Institute for the Study of Intellectual Behavior, University of Colorado, and is Publication 87 of the Institute. The work was supported in part by NSF Grants BNS72-02084 and BNS77-06799 and was performed using the facilities of the Computer Laboratory for Instruction in Psychological Research, which is supported in part by the University of Colorado. The second author was supported by a postgraduate scholarship from the National Research Council of Canada. A preliminary report of Experiment 1 was presented at the Psychonomic Society Meeting, Denver, Colorado, November 1975. Requests for reprints should be sent to Peter G. Polson, Department of Psychology, University of Colorado, Boulder, Colorado 80309. study nor the Atwood and Polson study directly tests the basic assumption of this framework that subjects use only information about the current problem state and its immediate successors to select a next move. This assertion is in direct contradiction to proposals that have been made by other investigators concerning process models for these types of problems. Egan and Greeno (1974), Greeno (1974), and Thomas (1974) have all claimed that move selection involves the planning and execution of multistep move sequences.

We begin with a description of the water jug task and a discussion of planning in the context of this task. Next, we present an extended version of the Atwood and Polson (1976) model developed by Atwood (1976). We then discuss the rationale underlying our experimental manipulations and present the observed results. Finally, we will discuss comparisons between observed performance and our model's predictions and alternative models that incorporate planning assumptions.

\section{The Task}

The water jug problems used in these studies are similar to those used by Luchins (1942) in his experiments on rigid behavior. The principal differences are that Luchins' tasks involved three operators ("fill-jug," "empty-jug," and "pour"), whereas our tasks involve only a single operator ("pour"), and our tasks require a greater number of moves to reach a solution. Our experiments employed a task with three water jugs (A, B, C) of varying sizes; for example, in the $(8,5,3)$ problem, the $\mathbf{A}$ jug has a capacity of 8 units, the $B$ jug 5 units, and the $C$ jug 3 units. Initially, the largest jug is full and the two smaller jugs are empty. The subject's task is to determine a series of moves that would divide 
the initial contents of the largest jug (A) evenly between the two largest jugs, $A$ and $B$. Water is transferred until the jug the subject is pouring from is emptied or the jug being poured into is filled. The jugs are not graduated, and water cannot be added or deleted during the course of solving the problem.

A graph of the $(8,5,3)$ problem is shown in Figure 1. The other problems used in these studies had very similar graphs. All have the same configuration of states at the top of the graph and two different solution paths. They differ in the length of the solution paths and other characteristics listed in Table 1.

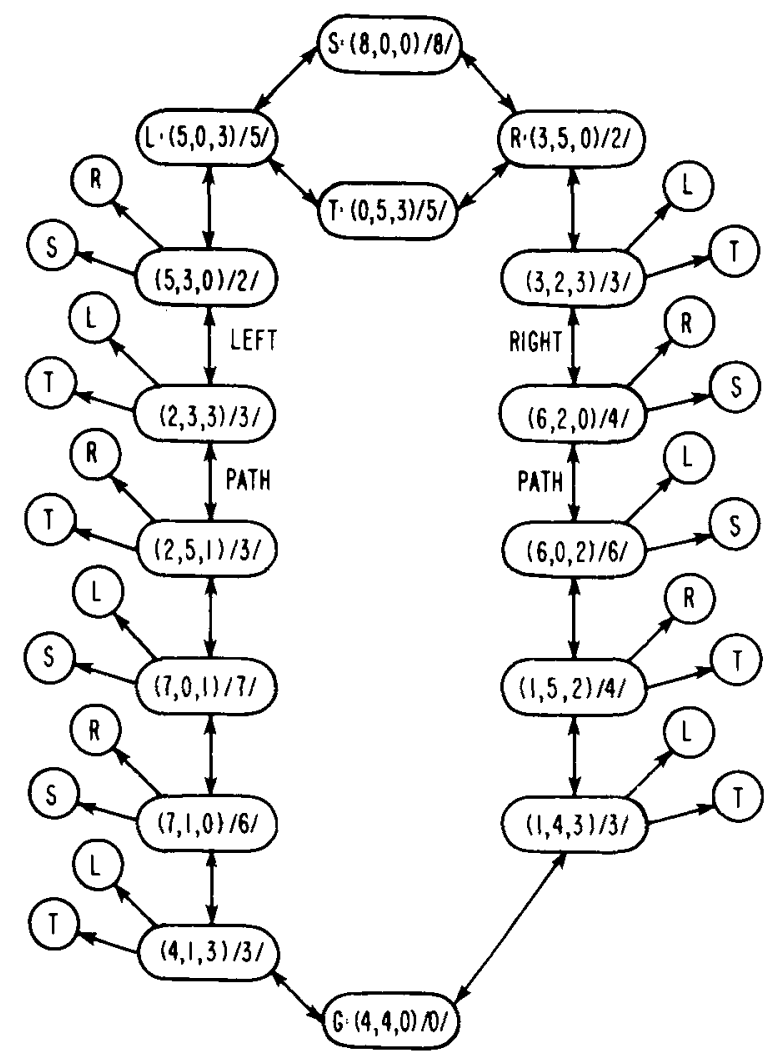

Figure 1. Graph of the possible states and legal moves for the $(8,5,3)$ problem. For each state, the three numbers in parentheses are the current content of Jugs A, B, C, respectively. S, T, etc., are the state labels.
State $S$ is the start state; the largest jug is full, and $B$ and $C$ are empty. From $S$, a subject has a choice of two moves. Pouring A into B leads to State R, the top of the right solution path. Pouring $A$ into $C$ leads to State L, the top of the left solution path. From both States $R$ and $L$, there are three possible moves: (1) a reversible forward move down the respective solution path, (2) a reversible move to the transition state, $T$, and (3) a reversible backward move to State $S$, the start state. The remaining states of the problem are on either one of the two solution paths. There are four possible moves from each state: a reversible forward move, a reversible backward move, and two irreversible moves to the top of the graph, States $\mathrm{S}, \mathrm{T}, \mathrm{R}$, and $\mathrm{L}$. We will refer to these irreversible moves as "looping moves."

Various aspects of the problems used in our two studies are presented in Table 1 . This table shows the solution path lengths, the number of violations of the means-ends move-selection heuristic, the number of looping moves, and irreversible moves back to States $\mathrm{S}$, $\mathrm{T}, \mathrm{R}$, and $\mathrm{L}$ for each problem. In addition, we show the number of looping moves that are classified as acceptable by our means-ends heuristic.

The theoretical and empirical analyses to be presented later will focus on the looping moves. Let us define a "correct" move as a forward move that leads to a new state. Reversible backward moves comprise only about $10 \%$ of the data, and we will ignore them in this discussion. We let "errors" be the looping moves (i.e., irreversible moves to states at the top of the graph). Observe that if a subject makes no errors, he will make a series of consecutive forward moves down one solution path or make a minor detour through State T. The modal subject's performance is some number of "errors" that are separated by varying numbers of consecutive successes terminated by a run of successes that leads to the goal state. Errors, number of looping moves, are thus a less variable measure of problem difficulty than total moves to solution and are not contaminated by lengths of the two solution paths.

\section{The Model}

In this section, we describe the process model for the water jug task that has been developed by Atwood

Table 1

Descriptions of the Experimental Problems

\begin{tabular}{|c|c|c|c|c|c|c|c|c|}
\hline & \multicolumn{8}{|c|}{ Problem } \\
\hline & \multicolumn{4}{|c|}{ Right Path } & \multicolumn{4}{|c|}{ Left Path } \\
\hline & $(8,5,3)$ & $(12,7,4)$ & $(10,7,3)$ & $(16,10,3)$ & $(8,5,3)$ & $(12,7,4)$ & $(10,7,3)$ & $(16,10,3)$ \\
\hline Length & 7 & 7 & 9 & 13 & 8 & 14 & 10 & 14 \\
\hline Number of Violations & 3 & 3 & 3 & 5 & 2 & 3 & 3 & 3 \\
\hline Possible Loops* & $2,3,2,3$ & $2,3,2,3$ & $3,4,4,4$ & $4,5,4,5$ & $3,3,3,3$ & $6,6,6,6$ & $4,4,4,5$ & $6,6,6,6$ \\
\hline Acceptable Looping Moves $\nmid$ & $0,1,2,1$ & $0,1,2,1$ & $0,0,2,1$ & $1,1,3,1$ & $1,0,3,1$ & $1,0,5,2$ & $0,0,3,1$ & $0,3,5,1$ \\
\hline
\end{tabular}

*Number of possible loops to States $S, T, R$, and $L$. †Number of acceptable looping moves to $S, T, R$, and $L$. 
(1976) and Atwood and Polson (1976). Our objective is to motivate our experimental procedures and the discussion of our results in terms of the model. The reader is referred to the earlier papers for detailed descriptions of the rationale behind the theory, reviews of related work, and other supporting evidence.

The model assumes that move selection in the water jug task involves the interaction of two sets of processes: evaluation processes and memory processes. The evaluation processes describe how means-ends move-selection heuristics and information retrieved from long-term memory (LTM) specify the evaluation of a move under consideration. The memory processes describe the storage and retrieval of information used in the selection of moves. A three-stage process model describes how the outputs of the memory and evaluation processes are combined to select a next move. It is assumed that subjects use only information about the current state and its immediate successors to select a next move. Alternative models incorporating planning assumptions are discussed later.

The evaluation processes. There are three aspects of the evaluation processes: (1) state evaluation, (2) an acceptability criterion, and (3) optimal move selection.

There are two components of the state-evaluation process: (1) the means-ends component and (2) the frequency component. Let $C_{i}(A)$ be the number of units of water in Jug $A$ for the ith state; let $G(A)$ be the number of units of water in $A$ specified by the goal (G) state. We define $C_{i}(B)$ and $G(B)$ similarly. The means-ends component of the evaluation function is $\left|C_{i}(A)-G(A)\right|+\left|C_{i}(B)-G(B)\right|$. The frequency component, which is similar to that proposed by Hintzman (1969) and others, incorporates information concerning the number of previous entries into State i into the evaluation process. Thus, the evaluation is modified by frequency information. Let $f_{i}$ be the frequency of entries into State i stored in LTM. Let a be the base and $t$ be the threshold. Further, let $\ln \left(f_{i}-t\right)$ equal 0 for all values of $f_{i} \leqslant t$. The frequency component equals $a \ln \left(f_{i}-t\right)$. The evaluation of State $i\left(e_{i}\right)$ is just the sum of the frequency and means-ends components.

We assume that subjects use the above evaluation function to classify moves as either acceptable or unacceptable. A subject compares the evaluation of his current state $\left(\mathrm{e}_{\mathbf{j}}\right)$ with the evaluation of the state resulting from the move under consideration $\left(\mathrm{e}_{\mathrm{j}}\right)$; he is indifferent to small differences in the values of the evaluation function and will consider a move to be acceptable if $e_{j}-e_{i}<\delta$. Any other move is unacceptable; that is, moves that lead to states with significantly higher values of the evaluation function are unacceptable.

Memory processes. Atwood and Polson (1976) assume that the memory structure and processes can be described by a simplified version of a multistore model for memory (Bower, 1975). Information about the current state and its successors is stored in short-term memory (STM) during the move-selection process. Information about previously visited states, which is utilized in the state-evaluation and move-selection processes, is stored in LTM.

During the first stage of the move-selection process, the subject computes and stores in STM the following information about the successor of the current state: (1) the move, (2) the resulting state, (3) its evaluation, and (4) information about the frequency of previous entries into this successor of the current state. Accurate information about at most $r$ moves can be stored in STM. We assume that information about various successors will be lost if the current state has more than $r$ successors.

The representations of states actually entered in the course of solving the problem are stored in LTM. Upon entry into a state, a subject increments by 1 a counter, $f_{i}$, with probability $s$. Information about previous entries into a state, $f_{i}$, is retrieved using a recognition process. "Old" states will always be recognized; "new" states are defined as those states for which $f_{i}=0$.

The move-selection process. The move-selection model describes how information from the evaluation processes and the memory processes are combined to select a next move.

During Stage 1, a subject randomly selects and evaluates each move without replacement. This process continues until the subject selects a move or until all moves are evaluated and rejected. The following decision rules are employed in Stage 1 (Atwood \& Polson, 1976, p. 197): (1) An unacceptable move is never taken. (2) The move leading to the immediately preceding state is never taken. (3) A move leading to the goal state is always taken. (4) An acceptable move leading to a new state is taken with probability $\alpha$. (5) An acceptable move leading to an old state is taken with probability $\beta$.

During Stage 2, a subject attempts to find a successor that has not been entered during a previous episode, $\mathrm{f}_{\mathrm{i}}=0$. A subject randomly selects a move if there is more than one such state. A subject enters Stage 3 only if there are no new successors.

During Stage 3, a subject attempts to select an optimal move on the basis of information generated during the execution of the Stage 1 process. If the current state has more than $r$ successors, the information necessary to select an optimal move will have been lost from STM, and the subject will randomly select any of the possible successors of this state. If the current state has $r$ or fewer successors, the subject will select the move that leads to the state with the lowest value of the evaluation function with probability $\alpha$. Otherwise, he randomly selects a move.

In order to account for the overall performance of subjects, the structure of water jug problems requires that we accurately predict the number of times subjects return to the start state, S. Several studies (e.g., Newell \& Simon, 1972, Chapter 7) indicate that returning to the 
start state of a problem is fairly common in human problem solving. Postexperimental interviews with subjects in the Atwood and Polson (1976) study sug. gested that at least some of these moves were made for the purpose of starting the problem over, a "desperation" move. Such a process has been incorporated into Stage 3 of the model. It is assumed that a subject in this stage will elect to return to the start state (if this is a legal transition) with probability $g$ before attempting to select an optimal move.

\section{Rationale for the Experiments}

Atwood (1976) and Atwood and Polson (1976) have shown that this model provides an excellent quantitative account of subjects' performance on various versions of the water jug task. A primary assumption underlying this model is that STM limitations prevent subjects from using more complex planning strategies and that moves are selected on the basis of information about the current state and its successors. Another possible interpretation of Atwood and Polson's results is that subjects are attempting to use more complex move-selection heuristics but fail to employ them consistently because of resource limitations. For example, consider the memory and other resource requirements for move generation. Atwood and Polson's subjects had to mentally calculate the successor resulting from each possible move, as well as perform evaluation and selection operations. If we assume that move generation uses a significant portion of subjects' resources, it may not be possible for them to reliably execute more complex planning strategies.

In the experimental conditions of the studies to be described later, subjects were given each possible move and its resulting state. In other conditions, subjects were told which states they had been in during previous episodes. If the arguments about resource allocation presented in the preceding paragraph are correct, we would expect that subjects would be able to execute more complex move-selection strategies when given a list of possible moves and their resulting states. The question then becomes, will subjects use more complex strategies? We argued earlier that various types of forward planning strategies would enable subjects to detect and eliminate looping moves. This would lead to a dramatic improvement in performance, especially in more difficult problems in which there are more looping moves and such moves cause subjects to have to retrace a longer solution path. For example, a large reduction in the number of looping moves should lead to a significant number of subjects solving the most difficult problem in a minimum number of steps.

The experiments to be reported in this paper compare performance on several water jug problems varying in difficulty in three different experimental conditions. Subjects in the control conditions were required to mentally calculate the results of each possible move, as well as to evaluate and select a next move. The display for these subjects is shown in Figure 2a. This is the same procedure used by Atwood and Polson (1976). Subjects in the move-availability conditions received the same information as subjects in the control conditions plus a list of all legal moves and their resulting states. The display for these subjects is shown in Figure $2 b$. The possible moves were displayed in a different random order for each move and successive entries into the same state. Subjects in the memory conditions received the same display as subjects in the move-availability conditions plus information about previously occupied states. For half the subjects in the memory conditions, an asterisk appears alongside the resulting state, indicating that the subject had previously been in this state. For the other half of the subjects, the asterisk indicated a new state.

Earlier, we argued that forward planning should lead to a dramatic facilitation of performance in both moveavailability and memory conditions. Further, there could be an interaction with problem difficulty, with more facilitation being observed in more difficult problems.

Explicit predictions can be derived from the Atwood and Polson (1976) model for the effects of the move-

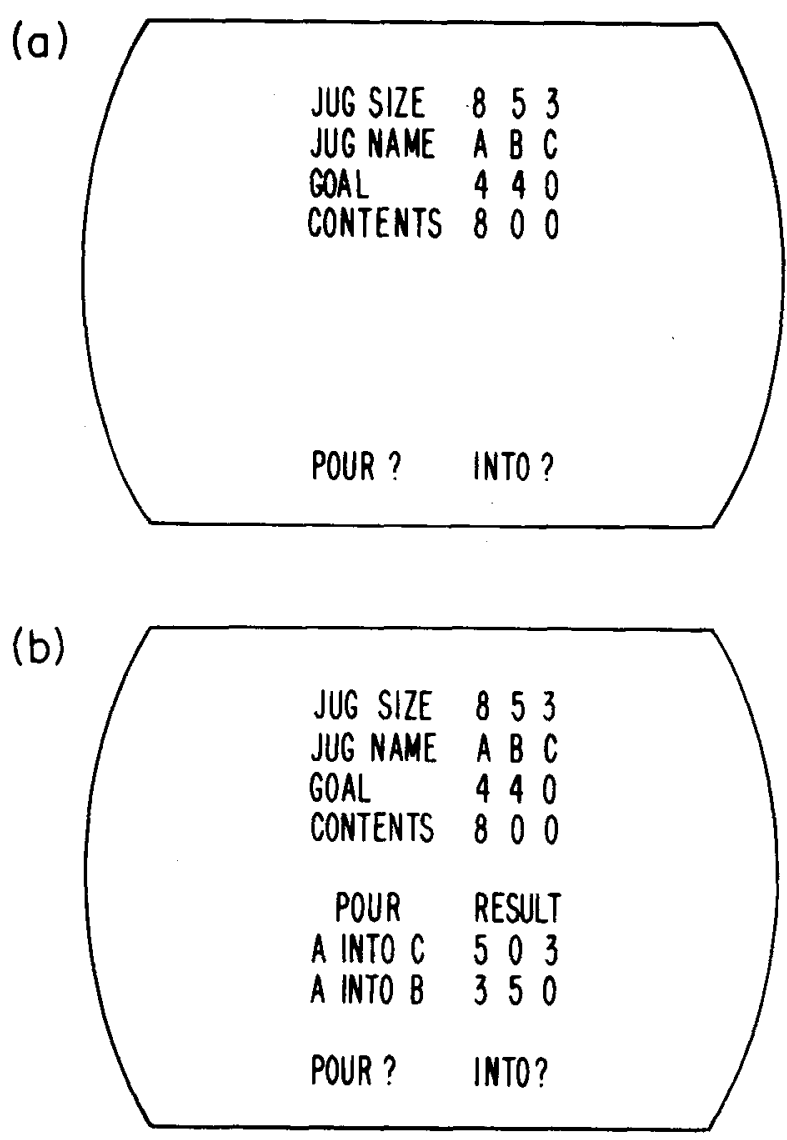

Figure 2. The display presented to subjects in the control (a) and move availability (b) condition. The information shown is for State $S$ of the $(8,5,3)$ problem. 
availability and memory conditions on performance. Following Newell and Simon (1972), we will assume that information in the display presented to subjects functions as an external memory and extends the capacity of STM. Recall that we assume that the move, the resulting state, its evaluation, and $f_{\mathbf{i}}$, the frequency of past entries into the state, are stored in STM. The parameter $r$ is the number of successors that can be reliably stored in STM. Presenting the moves and their resulting states on the display effectively increases the capacity of STM. Thus, we assume $r=4$ in the moveavailability and memory conditions, which is the maximum number of successors in these problems.

Predictions for $\mathrm{s}$, the probability of storing a representation of the current state in LTM, are not so clearcut. We will assume that the move-availability condition does not affect the value of $s$, and thus, the observed values should be equal for control and move-availability conditions. We assume s equals 1.0 in the memory conditions; the information to be retrieved from LTM, old vs. new, is displayed on the screen.

The move-availability and memory conditions will have very small effects on performance, if our experimental manipulations only affect $\mathrm{r}$ and $\mathrm{s}$, the memory parameters.

\section{Planning}

The model described in the previous section assumes that subjects use only information about the current state and its immediate successors to select a move. That is, we assume that naive subjects are not capable of any form of planning behavior. In this section, we will briefly outline what form alternative models that incorporate planning mechanisms might take. We will not consider planning models in general but will restrict this discussion to the types of planning that might occur in water jug problems.

Since our model incorporates some of the mechanisms employed by the General Problem Solver (GPS, Ernst \& Newell, 1969), it is reasonable to consider planning by abstraction, which Newell and Simon (1972, pp. 428-435) suggest as a planning heuristic for GPS. These authors are correct in noting that "This procedure acts as an antidote to the limitation of means-ends analysis in seeing only one step ahead" (Newell \& Simon, 1972, p. 428). This planning heuristic involves reformulating the problem in such a way that certain details of the problem states and operators that can be applied to these states are omitted. Since the task used here involves a single operator and rather simply structured problem states, quite unlike the logic problems considered by Newell and Simon, we see no reasonable way to plan by abstraction in this task.

A second type of planning involves the generation of subgoals. With this heuristic, a subject would identify a "stepping-stone state" that must be reached in order to achieve a solution and attempt to achieve that state before achieving the goal state. For illustration, we will consider a subgoal that would be useful in solving the $(8,5,3)$ problem. In this problem, the initial state is $(8,0,0)$, and the goal state is $(4,4,0)$. In order to solve this problem, it is necessary to reach some problem state in which one jug contains a single unit. The smallest jug, with a capacity of 3 , could then be added to produce the goal state.

Assume, for example, that a subject selected as a subgoal the state $(7,0,1)$. Such a selection would lead to behavior that we do not observe in our data. Using the means-ends evaluation function described earlier, the initial evaluations of problem states would be changed. In this case, the start state (S) would have an evaluation of $1, R$ of $9, L$ of 2 , and $T$ of 12 . This would lead to a prediction of a much larger number of looping moves to States $S$ and $L$ and a smaller number to $R$ than are observed.

In general, subgoaling involves the identification of one or more intermediate states on the solution path, stepping-stone states. Achieving each one of the steppingstone states in the proper sequence defines a series of subproblems whose collective solutions are much simpler, in the sense that there are fewer solution paths, than the solution to the original problem. We do not think that this is a reasonable type of planning mechanism for the water jug task. We have not been able to discover invariant properties of states on a solution path that would enable an individual to identify stepping-stone states. GPS (Ernst \& Newell, 1969) attempts to use meansends analysis to identify stepping-stone states. This heuristic does not work for water jug problems, and GPS has a difficult time with the task.

A third type of planning that could be used in this task involves $n$-move look-ahead or planning an n-move sequence. Such a process has been proposed for rivercrossing problems (Greeno, 1974) and for transformation problems in general (Greeno, 1978, p. 248). Like planning by creating subgoals, however, such a heuristic would alter the means-ends evaluations assigned to moves and, as a result, produce move patterns unlike those observed. There would be a very different pattern of acceptable and unacceptable moves for each state if a subject was selecting moves on the basis of the evaluations of states $\mathrm{n}$ steps from his current position.

Move-selection processes based on information obtained by some type of n-move look-ahead would be forced to make unreasonable assumptions about STM limits. There are four possible moves from each state except for the states at the top of the graph (S, T, R, and $\mathrm{L}$ ). We feel that calculating moves, evaluations, and retaining information about four or more move sequences would exceed the limits of STM.

On the basis of the preceding discussion, we conclude that any planning model that involves an alteration to the type of means-ends evaluation heuristic employed here would predict different patterns of move choices 
from those we observe in this task. Any planning model for this task, therefore, must include something in addition to a means-ends heuristic. Greeno (1974), in considering what is learned in the course of repeatedly solving a problem, argues that subjects acquire global information about the structure of the task. Learning about the structure of the task appears to be the most viable planning model for this task domain.

Given the structure of the water jug task, the most useful global information that a subject could acquire is to learn that a large number of moves loop back to the start of the problem. This would suggest a strategy of avoiding moves that lead to these states. Notice that if a subject initially had this strategy, a minimum path or near minimum path solution would result. It is possible, however, that subjects could acquire this strategy during the course of problem solving. That is, we assume that subjects initially approach a problem with only the processes incorporated into our model, but on each trial with some probability $\mathrm{p}$, they learn to avoid looping moves and, consequently, solve the problem in the minimum number of moves from the current problem state. The degree to which subjects are capable of planning is, therefore, a function of the parameter $p$. If a great deal of planning occurs, this parameter should be near one; if no planning occurs, it should be very near zero.

If our model for the water jug task is correct, then it should be able to account for performance in the control and both experimental conditions. Any variations in parameter values across conditions should be interpretable in the context of the process assumptions of the model. It would be reasonable to assume that a majority of the parameters of the model will be constant across conditions and problems. Further, we should be able to fit the data from the two experimental conditions with the values of the memory parameters $r=4$ in the memory and move-availability conditions and $s=1.0$ in the memory conditions. If the subjects use more complex planning processes, then the moveavailability and memory conditions should lead to very large improvements in performance, and the model should not be able to fit the data from these conditions.

\section{EXPERIMENT 1}

\footnotetext{
Method

Design. There were two experimental conditions. In the control condition, subjects were required to mentally calculate and select possible moves; in the move-availability condition, subjects were provided a list of possible successor states. Three experimental problems were used. These problems were $(8,5,3)$, $(12,7,4)$, and $(16,10,3)$. Conditions and problems were factorially combined to produce a 2 by 3 between-subjects design.

Subjects. Subjects were recruited through a newspaper advertisement and were paid $\$ 2$ for participating in the experiment. Subjects were randomly assigned to experimental conditions and problems in the order of their arrival at the laboratory. Forty subjects were assigned to each condition and problem,
}

with the exception of the $(12,7,4)$ and $(16,10,3)$ problems of the move-availability condition, which involved 41 subjects.

The primary reason for including such a relatively large number of subjects in each cell is that a major objective of our research is to carry out computer simulations in an effort to obtain accurate quantitative fits to the observed data. Water jug problems, like many problem solving tasks, involve a great deal of variability in performance. Since our model predicts very small differences in performance as a function of experimental condition, it is necessary to have fairly stable performance statistics. Note also that in determining the goodness of fit between observed and predicted performance we are attempting to prove the null hypothesis that there are no differences. Increasing the degrees of freedom in our analysis results in a stronger test.

Apparatus. The execution of the experiment was controlled by a Xerox Sigma 3 computer. The problems were presented to the subject on a four-phase system CRT display terminal. The subject responded by pressing buttons mounted in a row; three buttons were labeled " $A$," " $B$," and " $C$ "; two buttons were labeled "DO IT" and "ERASE." Presentation of the problems and data recording were performed by a program written in FORTRAN IV

From one to six subjects were run concurrently under the control of the CLIPR/RBM operating system. The procedure was subject paced, and an independent sequence of events was presented to each subject. Each pair of terminals was in a small room off a large common room. The tables were positioned in each experimental room so that subjects faced different walls.

Procedure. On entering the experimental room, the subject was given a set of written instructions. The instructions contained a detailed description of the task, information about the content of the CRT display, and a description of how to respond. After having any questions answered by the experimenter, the subjects solved a practice problem (jug sizes of 6,4 , and 1 ). Subjects were then given the experimental problem. The problem was terminated if subjects failed to solve within 100 moves (not counting illegal or erased moves)

A common procedure was used for all problems. Each problem began with appropriate information, as shown in Figure 2, being presented on the CRT display. The line labeled "JUG SIZE" specified the sizes of the three jugs for the current problem; the "GOAL" line specified the respective goal state. The "CONTENTS" line changed appropriately after each move. In the move-availability condition the list of randomly ordered successor states was displayed below the "CONTENTS" line.

The subject entered a move by first pressing one of the buttons labeled " $A$," "B," or " $C$ " to indicate the jug he wished to pour from. The subject then pressed a second button to indicate the jug he wished to pour into. The "POUR? INTO?" line was changed appropriately to display the responses made by the subject after he had entered both responses. In the moveavailability condition, the list of successor states was also appropriately updated. The subject could erase an incorrectly input or undesirable move by pressing the "ERASE" button. Pressing "DO IT" caused the move to be made and the "CONTENTS" line (and list of successor states in the move-availability condition) to be changed appropriately. The message "ILLEGAL MOVE TRY AGAIN" was displayed for $2 \mathrm{sec}$ in the event the subject entered an illegal move (pouring from an empty jug or into a full jug). At the end of this time, this message was erased, and the message "POUR? INTO?" reappeared.

\section{Results}

The dependent variables of interest are total legal moves to solution and looping moves. These moves were defined as irreversible moves from one of the solution paths back to one of the states at the top of the solution 
Table 2

Observed (O) and Predicted (P) Means and Standard Deviations for Legal Moves to Solution and Observed and Predicted Means for Looping Moves to States at the Top of the Solution Graphs (Experiment 1)

\begin{tabular}{|c|c|c|c|c|c|c|c|c|}
\hline & \multicolumn{4}{|c|}{ Legal Moves } & \multicolumn{4}{|c|}{ Looping Moves } \\
\hline & \multicolumn{2}{|c|}{ Control } & \multicolumn{2}{|c|}{ Move Availability } & \multicolumn{2}{|c|}{ Control } & \multicolumn{2}{|c|}{ Move Availability } \\
\hline & 0 & $\mathbf{P}$ & 0 & $\mathbf{P}$ & 0 & $\mathbf{P}$ & 0 & $\mathbf{P}$ \\
\hline & \multicolumn{8}{|c|}{ Problem $(8,5,3)$} \\
\hline \multirow[t]{2}{*}{$\begin{array}{l}\text { Mean } \\
\text { SD }\end{array}$} & $\begin{array}{l}27.85 \\
24.06\end{array}$ & $\begin{array}{l}28.46 \\
26.48\end{array}$ & $\begin{array}{l}20.58 \\
18.60\end{array}$ & $\begin{array}{l}21.30 \\
17.36\end{array}$ & 4.45 & 4.96 & 2.27 & 2.98 \\
\hline & \multicolumn{8}{|c|}{ Problem $(12,7,4)$} \\
\hline \multirow[t]{2}{*}{$\begin{array}{l}\text { Mean } \\
\text { SD }\end{array}$} & $\begin{array}{l}36.28 \\
29.14\end{array}$ & $\begin{array}{l}35.54 \\
31.64\end{array}$ & $\begin{array}{l}33.15 \\
26.57\end{array}$ & $\begin{array}{l}31.92 \\
26.95\end{array}$ & 5.86 & 6.14 & 3.89 & 4.74 \\
\hline & \multicolumn{8}{|c|}{ Problem $(16,10,3)$} \\
\hline $\begin{array}{l}\text { Mean } \\
\text { SD }\end{array}$ & $\begin{array}{l}58.72 \\
31.53\end{array}$ & $\begin{array}{l}57.92 \\
35.13\end{array}$ & $\begin{array}{l}48.75 \\
32.18\end{array}$ & $\begin{array}{l}48.38 \\
32.94\end{array}$ & 8.90 & 10.04 & 6.29 & 5.81 \\
\hline
\end{tabular}

graph, States S, T, R, or L. Moves between these four states were physically possible but were not counted as looping moves. Only those moves taken from states further down the solution paths back to one of the top states were counted as looping moves. This type of move is clearly an error or backward step in terms of trying to solve a problem of the type used in these experiments, and it is of interest in testing our theoretical predictions.

Means and standard deviations of total legal moves and means of total looping moves for all conditions and problems are shown in Table 2 . One randomly selected subject from each of the problems $(12,7,4)$ and $(16,10,3)$ of the move-availability condition was dropped to simplify the data analyses. An analysis of variance of legal moves to solutions that included conditions and problems as between-subjects variables revealed a significant effect of problems $[F(2,234)=23.84, p<.001]$. The effect of conditions just failed to reach significance $[F(1,234)=3.68, p<.06]$, and there was no evidence of an interaction between conditions and problems $(\mathrm{F}<1)$. An analysis of total looping moves revealed that move-availability subjects made significantly fewer looping moves $[F(1,234)=9.60, p<.005]$. The frequency of looping moves varied as a function of problems $[F(2,234)=11.74, p<.001]$.

\section{Conclusions}

Providing information about successor states did not dramatically affect the number of legal moves required to reach a solution. However, when looping moves were analyzed, a reliable reduction in such moves was observed when the move-availability and control conditions were compared.

The number of legal moves required to reach a solution and the number of looping moves made during attempts to solve the problem are, of course, correlated. Our results suggest, however, that the number of looping moves may be a more sensitive measure of behavior in a water jug problem than total moves to solution.

\section{EXPERIMENT 2}

\section{Method}

Design. The experimental design involved two factorially combined between-subjects factors: conditions (three different levels of information) and problems (four different sets of jug sizes). The control and move-availability conditions were identical to those used in Experiment 1. The third level of information given in the present experiment included move-availability information supplemented by markers indicating which problem states the subject had previously entered. This is the memory condition. The problems were $(8,5,3),(12,7,4),(10,7,3)$, and $(16,10,3)$.

Subjects. The design was replicated over two populations of subjects. In the first case, subjects were 240 students at the University of Colorado participating in partial fulfillment of an introductory psychology course requirement. The second replication involved 240 subjects who were paid $\$ 2$ for their participation. In each replication, 20 subjects were randomly assigned to each of the 12 combinations of conditions and problems.

Apparatus and Procedure. The equipment and procedure used in Experiment 1 were also used in the present experiment. In the memory conditions, the display was the same as that in Figure 2b, except that moves leading to states (sets of jug contents) that the subject had already entered were specially marked. For half of the subjects in the memory condition, previously visited states were marked with an asterisk; for the remaining subjects, states that had not yet been visited were marked with an asterisk. Therefore, the display provided subjects with accurate information about whether or not they had previously visited any of the states that they could legally reach within one move.

\section{Results}

The manner in which previously visited states were identified in the memory condition (marking previously visited states vs. marking states not yet visited) did not affect the number of legal moves required by memory condition subjects to solve a problem. Hence, the following analyses were done by collapsing across the two methods of providing memory information.

Table 3 shows the means and standard deviations of total legal moves to solution and the means for total 
Table 3

Observed (O) and Predicted (P) Means and Standard Deviations for Legal Moves to Solution and Observed and Predicted Means for Looping Moves to States at the Top of the Solution Graphs (Experiment 2)

\begin{tabular}{|c|c|c|c|c|c|c|c|c|c|c|c|c|}
\hline & \multicolumn{6}{|c|}{ Legal Moves } & \multicolumn{6}{|c|}{ Looping Moves } \\
\hline & \multicolumn{2}{|c|}{ Control } & \multicolumn{2}{|c|}{ Move Availability } & \multicolumn{2}{|c|}{ Memory } & \multicolumn{2}{|c|}{ Control } & \multicolumn{2}{|c|}{ Move Availability } & \multicolumn{2}{|c|}{ Memory } \\
\hline & 0 & $\mathrm{P}$ & 0 & $\mathbf{P}$ & 0 & $\mathbf{P}$ & $\mathrm{O}$ & $\mathbf{P}$ & 0 & $\mathbf{P}$ & 0 & $\mathbf{P}$ \\
\hline & \multicolumn{12}{|c|}{ Problem $(8,5,3)$} \\
\hline \multirow[t]{2}{*}{$\begin{array}{l}\text { Mean } \\
\text { SD }\end{array}$} & $\begin{array}{l}34.50 \\
29.09\end{array}$ & $\begin{array}{l}33.42 \\
27.64\end{array}$ & $\begin{array}{l}21.80 \\
19.84\end{array}$ & $\begin{array}{l}22.53 \\
18.24\end{array}$ & $\begin{array}{l}20.73 \\
20.00\end{array}$ & $\begin{array}{l}21.49 \\
18.10\end{array}$ & 6.10 & 6.24 & 2.98 & 3.50 & 2.72 & 2.94 \\
\hline & \multicolumn{12}{|c|}{ Problem $(12,7,4)$} \\
\hline \multirow[t]{2}{*}{$\begin{array}{l}\text { Mean } \\
\text { SD }\end{array}$} & $\begin{array}{l}48.60 \\
29.06\end{array}$ & $\begin{array}{l}45.21 \\
34.50\end{array}$ & $\begin{array}{l}28.18 \\
21.04\end{array}$ & $\begin{array}{l}30.16 \\
24.53\end{array}$ & $\begin{array}{l}26.85 \\
21.77\end{array}$ & $\begin{array}{l}26.41 \\
22.16\end{array}$ & 8.28 & 8.36 & 3.95 & 4.35 & 3.00 & 3.38 \\
\hline & \multicolumn{12}{|c|}{ Problem 10,7,3) } \\
\hline $\begin{array}{l}\text { Mean } \\
\text { SD }\end{array}$ & $\begin{array}{l}38.30 \\
26.13\end{array}$ & $\begin{array}{l}39.56 \\
33.06\end{array}$ & $\begin{array}{l}31.60 \\
26.09\end{array}$ & $\begin{array}{l}32.49 \\
27.40\end{array}$ & $\begin{array}{l}27.60 \\
21.56\end{array}$ & $\begin{array}{l}28.35 \\
25.39\end{array}$ & 5.65 & 6.76 & 3.45 & 4.03 & 3.18 & 3.32 \\
\hline & \multicolumn{12}{|c|}{ Problem $(16,10,3)$} \\
\hline $\begin{array}{l}\text { Mean } \\
\text { SD } \\
\end{array}$ & $\begin{array}{l}65.00 \\
31.36 \\
\end{array}$ & $\begin{array}{l}64.76 \\
33.79\end{array}$ & $\begin{array}{l}58.52 \\
34.20\end{array}$ & $\begin{array}{l}57.50 \\
32.04\end{array}$ & $\begin{array}{l}43.25 \\
31.39\end{array}$ & $\begin{array}{l}43.11 \\
29.54 \\
\end{array}$ & 10.18 & 11.52 & 7.66 & 7.43 & 4.90 & 5.11 \\
\hline
\end{tabular}

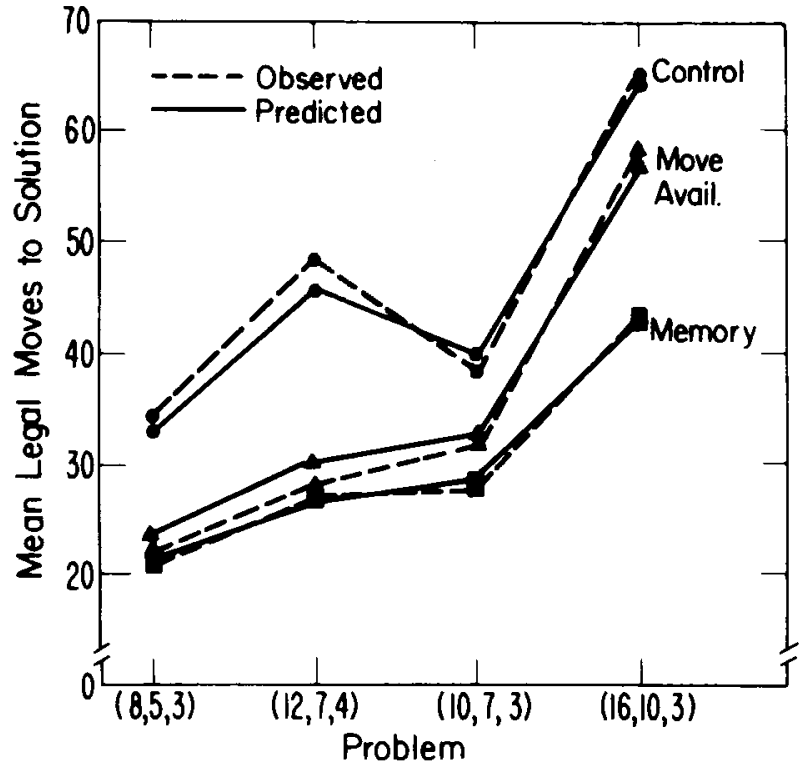

Figure 3. Observed and predicted values of legal moves to solution in Experiment 2.

looping moves as a function of condition and problem. The mean legal moves are also displayed in Figure 3. In general, the paid subjects required fewer legal moves to solve $[F(1,456)=6.37, p<.02]$. Since there were no significant interactions involving the replication variable, these two subject populations were pooled for purposes of statistical analysis and computer simulation.

The analysis of legal moves to solution also revealed a large effect of problems $[F(3,456)=28.83, p<.001]$. The different levels of information also had an effect on legal moves to solution $[F(2,456)=17.39, p<.001]$. According to a Tukey A test, subjects in the control condition required significantly $(\mathrm{ps}<.01)$ more moves to solve a problem than did move-availability or memory subjects, who did not reliably differ from each other. Conditions and problems did not interact.

The data for total looping moves were collapsed across replications and were analyzed with conditions and problems as between-subjects variables. The different conditions resulted in reliably different numbers of total looping moves $[F(2,468)=25.75, p<.001]$. All three conditions differed from each other (Tukey A, ps $<.01$ ), with subjects in the control condition making the most looping moves and subjects in the memory condition making the fewest. The problems also differed in the number of looping moves that subjects made while solving them $[F(3,468)=12.06, p<.001]$

\section{Conclusions}

In Experiment 1, the effects of the move-availability condition on legal moves to solution just barely failed to reach statistical significance. In Experiment 2, there was an effect due to providing information about the legal successors to the current problem state (move-availability) and a very small, nonsignificant further improvement due to adding information about which problem states had previously been visited. Although statistically very significant, these effects were not as dramatic as would be expected, if forward planning was an important problem solving mechanism in this condition. In the control condition, the mean legal moves to solution were approximately five times the minimum number of moves required to reach a solution, and in the moveavailability and memory conditions, subjects still required three or four times the minimum number of moves to reach a solution.

\section{SIMULATIONS}

Our next objective is to show that the model described in the introduction can provide an adequate account of 
the experimental results from the two studies that we have reported. We feel that the notion of adequate account in this context has two senses. First, the model obviously must provide an adequate description of the data from all of the experimental conditions in both experiments. Second, the parameter estimates that are required to fit the data in the experimental conditions must themselves be consistent with the predictions that were made earlier. We begin this section by summarizing the model in terms of its free parameters. We then go on to briefly describe the procedures used to obtain best-fitting parameters and the goodness-of-fit results.

Our model of performance in the water jug task has three sets of free parameters. The first set describes the problem solving processes $(\alpha$, the probability of taking an acceptable means-ends move to a new state; $\delta$, the indifference criterion; and $\mathrm{g}$, the probability of making a move to the start state from Stage 3). The second set characterizes the memory process ( $s$, the encoding parameter; base, $a$, and threshold, $t$, the two parameters of the frequency process; and $r$, the size of STM). The third set is a single parameter that characterizes the interaction between problem solving and memory processes $(\beta$, the probability of taking an acceptable move to an "old" state).

The details of the methods used to estimate parameters are given in Atwood and Polson (1976) and Jeffries et al. (1977). In summary, a search process was used to find a best-fitting set of parameter values for various collections of groups from each experiment. Following Atwood and Polson (1976), we assume that none of the parameters varied as a function of water jug problem type. For each experiment, a single set of trial parameters for a given experimental condition was evaluated by having our simulation program generate and process data for 250 simulated subjects on each of the water jug problems. Various summary statistics were computed for each simulated data set, and then the values of these statistics were compared with their corresponding observed values. A search terminated when a set of parameters was found that minimized the differences between observed and predicted values of the summary statistics averaged across the three or four groups defined by variations in water jug problems.

The model was fit to the data from each experiment using the following procedure. We first found a bestfitting set of parameters for the control groups using the procedures outlined in the preceding paragraphs. Once we had found parameters that provided adequate fits for the control conditions, an initial attempt was made to fit the data from the move-availability and memory conditions. Earlier, we conjectured that the display presented to subjects in the move-availability and memory conditions would augment the size of STM. Accordingly, for these conditions, $r$ was set to 4 , the maximum number of moves from any state in this type of water jug problem. We further conjectured that $\mathrm{s}$, the encoding parameter, would equal 1.0 in the memory condition because information concerning the old/new distinction is presented to the subject in the display. Finally, adjustments were made in $\beta$ to get the best possible fits to data from the move-availability and memory conditions. All other values of the parameters were fixed across experiments, problems, and experimental conditions. We selected parameter $\beta$ for finetuning manipulations because this parameter describes how problem solving and memory processes interact. An experimental manipulation that facilitates memory processes, therefore, should affect this parameter. It would be expected that such facilitation would result in a decrease in this parameter. Recall that $\beta$ is the probability of making a means-ends move to a state that the subject recognizes as being "old."

The best-fitting parameter values for each of the experimental conditions in both experiments is given in Table 4 . The observed and predicted means and standard deviations for legal moves and total looping moves are given in Table 2 for Experiment 1 and in Table 3 for Experiment 2. Figure 3 shows the mean observed and predicted legal moves for Experiment 2, and Figure 4 gives the observed and predicted looping moves to each of the top four states. Evaluation of goodness of fit was done by using $F$ tests to compare observed and predicted means and variances and by the graphical comparison of numerous other summary statistics. The reader is referred to Atwood and Polson (1976) for a more detailed discussion of the evaluation of comparisons between observed and predicted performance in this paradigm. In Experiment 1, the $F$ ratios comparing observed and predicted means were all less than 1.0. The observed $F$ ratios for variances all had $p$ values greater than .10 . In Experiment 2, we again found equally good fits to the observed means. However, there was a significant difference between observed and predicted variance in one cell of the design [control condition, problem $(10,7,3)$, $\mathrm{F}(249,39)=1.60, \quad .025<\mathrm{p}<.05]$. Examination of Figure 4 shows that there was excellent correspondence between observed and predicted looping moves in practically all experimental conditions. The structure of

Table 4

Best-Fitting Parameter Values for Each of the Experimental Conditions in Both Experiments

\begin{tabular}{lcccccccc}
\hline \multirow{1}{*}{$\begin{array}{c}\text { Condi- } \\
\text { tion }\end{array}$} & $\alpha$ & $\beta$ & $\mathrm{s}$ & $\mathrm{r}$ & $\mathrm{g}$ & $\mathrm{a}$ & $\mathrm{t}$ & $\delta$ \\
\cline { 2 - 9 } & & \multicolumn{8}{c}{ Experiment 1} \\
Control & .60 & .17 & .90 & 3 & .05 & 3.5 & 3 & 1 \\
MA & .60 & .14 & .90 & 4 & .05 & 3.5 & 3 & 1 \\
& \multicolumn{8}{c}{ Experiment 2} \\
Control & .60 & .24 & .90 & 3 & .05 & 3.5 & 3 & 1 \\
MA & .60 & .16 & .90 & 4 & .05 & 3.5 & 3 & 1 \\
Memory & .60 & .14 & 1.00 & 4 & .05 & 3.5 & 3 & 1 \\
\hline
\end{tabular}

Note $-M A=$ move availability. 

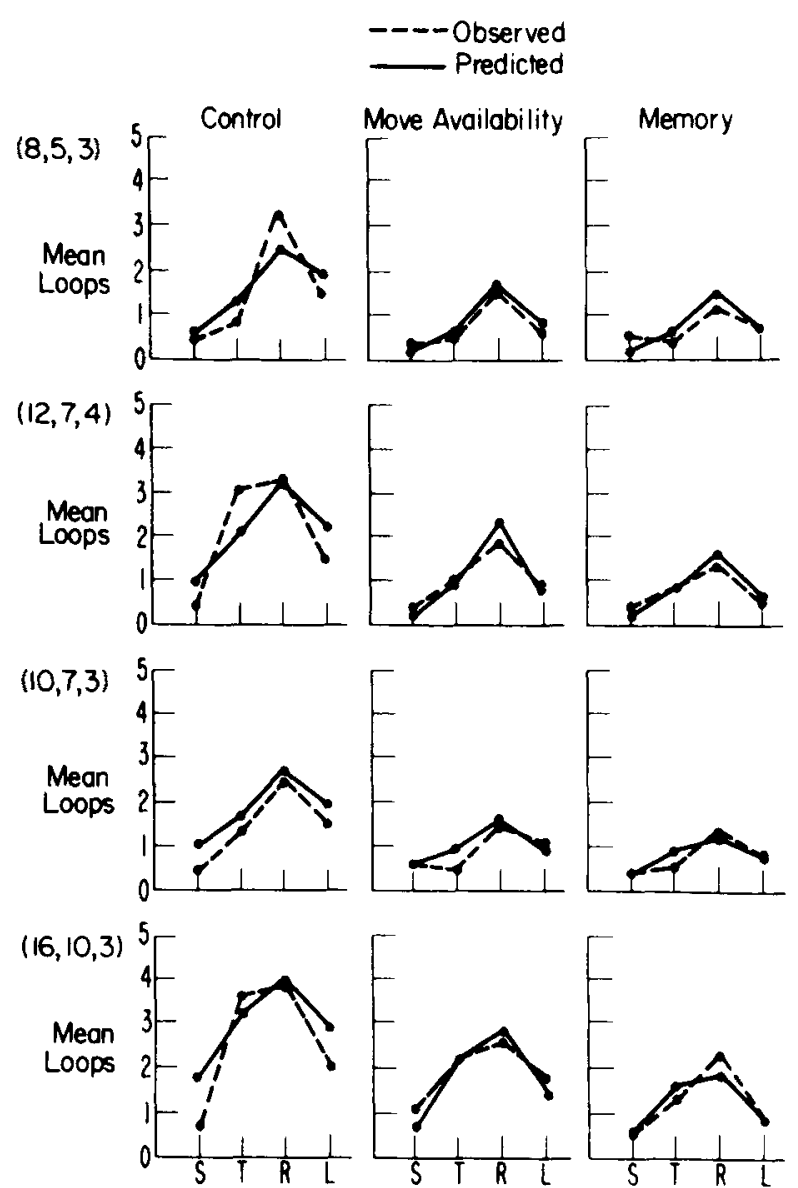

Figure 4. Observed and predicted number of looping moves to States S, T, R, and $\mathrm{L}$ in Experiment 2.

the water jug task is such that if one is able to account for total moves to solution and looping moves to the top states, one has accounted for a large majority of the variance in these data.

Examination of Table 4 shows that the goodness-offit results that we described in the preceding paragraph were obtained by manipulating only three parameters in order to account for six experimental conditions in Experiment 1 and 12 experimental conditions in Experiment 2 . The parameter $\beta$ was the only free parameter that was manipulated across the various experimental conditions in order to account for varying levels of performance. Differences between control, moveavailability, and memory conditions were accounted for by a mixture of a priori shifts in $r$ and $s$ and by manipulations of $\beta$. All parameters of the model were held constant across variations of problems. The only differences between experiments were described by minor shifts in the parameter $\beta$. Although the reader may feel that the model presented in this paper has a very large number of free parameters and is needlessly complex, we note that we are able to provide an excellent account of the data from experimental conditions that lead to wide variations of performance with very minor changes in the parameters of the model. On the other hand, if we had been required to make numerous ad hoc changes in the values of parameters in the model as a function of experiments, problems, and experimental conditions, the goodness-of-fit results would have been less impressive, and the inconsistencies in parameter values could have been interpreted as evidence against the theory. This, however, was not the case.

It may be the case, however, that the differences between the control, move-availability, and memory conditions are due to the fact that subjects are able to employ some type of planning strategy. In discussing alternative explanations of this data in terms of planning models, we will focus on the data from the memory condition of Experiment 2. This condition shows the largest reduction in looping moves, and comparisons between the memory and control conditions show the largest improvements in performance. We argue that if planning is a significant process in the water jug task, it should be most apparent in the memory condition.

In the introduction, we discussed three different classes of planning models. The first two assumed that a subject used some variety of n-move look-ahead, selecting moves on the basis of backed-up values, or that a subject chose an intermediate state as a subgoal and then defined the subproblem of achieving this intermediate state before going on to achieve the goal state. We argued that any model from either of these two classes would predict move patterns very different from those observed, assuming that a common evaluation process was used for all models. These differences in move patterns would be most apparent in the relative frequencies of looping moves. Examination of Figure 4, however, shows that the model proposed in this paper is able to account for patterns of looping moves for all experimental conditions. Thus, we conclude that any model that assumes n-move look-ahead or the generation of subgoals can be rejected.

We feel that the most viable candidate for a planning process in the water jug task is a model that assumes that a subject acquires knowledge about the structure of the problem. In particular, a subject learns to avoid any move that leads to one of the four states at the top of the problem graph: S, T, R, or L. We made the assumption that learning the structure of the problem was a type of insight process and that on each trial, with probability $\mathrm{p}$, a subject would discover this structure and then take only forward moves. If subjects were in States $\mathrm{S}$ or $\mathrm{T}$ when this insight occurred, a move to State $\mathrm{R}$ or $\mathrm{L}$ was randomly chosen, followed by a sequence of forward moves. Since the memory and move-availability conditions do lead to reductions in the numbers of looping moves, this seems to be a plausible way to implement planning in this task.

We evaluated this planning model by attempting to fit the data from the memory conditions of Experiment 2. The simulations were run using the parameters from the 
control condition, except for the parameters $\mathrm{r}$ and $\mathrm{s}$, which were set to 4 and 1.0 , respectively, as dictated by our assumptions concerning the effects of these experimental manipulations. Acceptable fits to observed performance measures were obtained by setting the planning parameter to .015 . For problems $(8,5,3)$, $(12,7,4),(10,7,3)$, and $(16,10,3)$, respectively, the observed (and predicted) legal moves to solution were 20.73 (20.97), 26.85 (26.56), 27.60 (27.27), and 43.25 (38.49). Predicted variances and looping moves statistics were similarly close to their respective observed values.

It seems that equally good fits to the data can be obtained by assuming either that the move-availability and memory conditions reduced the value of $\beta$, thus making looping moves less likely, or that the planning parameter is nonzero in these conditions. Observe, however, that the planning parameter has a very small value. In fact, in only one problem did a majority of the simulated subjects invoke the planning process.

The difficulty in discriminating between these two explanations is not surprising. Our original model required a reduction in the probability of taking an acceptable move to a state that had been previously entered. The planning model also reduces (to zero) the probability of taking moves to certain states. Since there is some overlap in the states affected, these models lead to similar predictions.

If planning does occur in this task, however, it appears to make a very small contribution to overall performance, given a planning parameter of .015 . More substantial values of this parameter, of around .1, result in predicted moves to solution that are only four or five moves greater than the minimum path solutions to these problems. This is a far higher level of performance than that observed. If, through other experimental manipulations, we could observe such levels of performance, we would consider such evidence as supporting the insight model of planning.

\section{Conclusions}

We argue that the model presented in this paper provides an excellent description of the data from the two experiments. The model correctly predicts the relative difficulties of the various problems and conditions and adequately describes the overall behavior of subjects in the process of solving the water jug problem.

We have argued that the experimental manipulations used in the studies described in this paper had the potential to produce very large facilitations of performance, especially for the more difficult problems. The information provided to subjects in the move-availability and memory conditions should have dramatically simplified any process that would enable the subject to detect and avoid looping moves. The avoidance of looping moves, especially in the more difficult problems like $(16,10,3)$, should have led to a dramatic facilitation of performance: an average of 17 legal moves to solution rather than the 43 moves to solution that was observed in the memory condition for the $(16,10,3)$ problem. Although the move-availability and memory conditions produced significant effects in terms of overall performance, these improvements were not as dramatic as one would have expected if subjects were capable of some form of planning in the water jug task. In all of the experimental conditions, we were able to simulate observed results by making reasonable and well-motivated parameter changes in a model that assumed no planning. Even if we incorporate a planning process, our results can only be modeled using a very small planning parameter, which makes a limited contribution to overall performance. Note that we were able to account for the data from all experimental conditions by making a priori changes in two of the memory parameters of the model and by manipulating one free parameter, which characterizes the interaction between problem solving and memory processes.

\section{REFERENCES}

Atwood, M. E. A theoretical analysis of behavior in a sequential problem solving task. Unpublished doctoral dissertation, University of Colorado, 1976.

Atwood, M. E., \& Polson, P. G. A process model for water jug problems. Cognitive Psychology, 1976, 8, 191-216.

BowE R, G. H. Cognitive psychology: An introduction. In W. K. Estes (Ed.), Handbook of learning and cognitive processes (Vol. 1). Hillsdale, N.J: Erlbaum, 1975.

Egan, D. E., \& GreEno, J. G. Theory of rule induction: Knowledge acquired in concept learning, serial pattern learning, and problem solving. In L. W. Gregg (Ed.), Knowledge and cognition. Potomac, Md: Erlbaum, 1974.

Ernst, G. W., \& Newel., A. GPS: $A$ case study in generality and problem solving. New York: Acadernic Press, 1969.

Greeno, J. G. Hobbits and orcs: Acquisition of a sequential concept. Cognitive Psychology, 1974, 6, 270-292.

Greeno, J. G. Natures of problem solving abilities. In W. K. Estes (Ed.), Handbook of learning and cognitive processes (Vol. 5). Hillsdale, N.J: Erlbaum, 1978.

Hintzman, D. L. Apparent frequency as a function of frequency and the spacing of repetitions. Journal of Experimental Psychology, 1969, 80, 139-145.

Jefries, R., Polson, P. G., Razran, L., \& Atwood, M. E. A process model for missionaries-cannibals and other rivercrossing problems. Cognitive Psychology, 1977, 9, 412-440.

Luchins, A. S. Mechanization in problem solving. Psychological Monographs, 1942, 54(Whole No. 248).

Newelt, A., \& Simon, H. A. Human problem solving. Englewood Cliffs, N.J: Prentice-Hall, 1972.

Thomas, J. C., JR. An analysis of behavior in the hobbits-orcs problem. Cognitive Psychology, 1974, 6, 257-269.

(Received for publication April 27, 1979; revision accepted December 10, 1979.) 